\title{
Can translocation help wild primates?
}

\author{
Julian Caldecott and Michael Kavanagh
}

Releasing animals into the wild, especially when they have become extinct there, is an appealing and dramatic conservation technique. It is, however, fraught with risks and often expensive; its success depends upon meticulous planning. The authors discuss the reasons for translocation, the many problems involved and the special cases where such an exercise may be justified.

In this paper, we examine some aspects of the use of translocation in the conservation and other management of non-human primates. Despite its superficial attractiveness, this technique is fraught with risks, and it is our aim to draw the attention of anyone contemplating a translocation programme to these risks and to suggest how they may be reduced or overcome. These guidelines were prepared at the request of the Conservation Working Party of the Primate Society of Great Britain and carry their endorsement; they are intended to complement discussions of related interest summarised elsewhere (Anon., 1968, 1979; Campbell, 1980; Grahame, 1980).

\section{What is translocation?}

It is the release in a new location of one or more free-ranging animals that come from anywhere other than the place in which they are released.

\section{Why translocate animals?}

\subsection{Conservation reasons}

2.1.1 A wild population may be threatened with local extinction by habitat destruction or for some

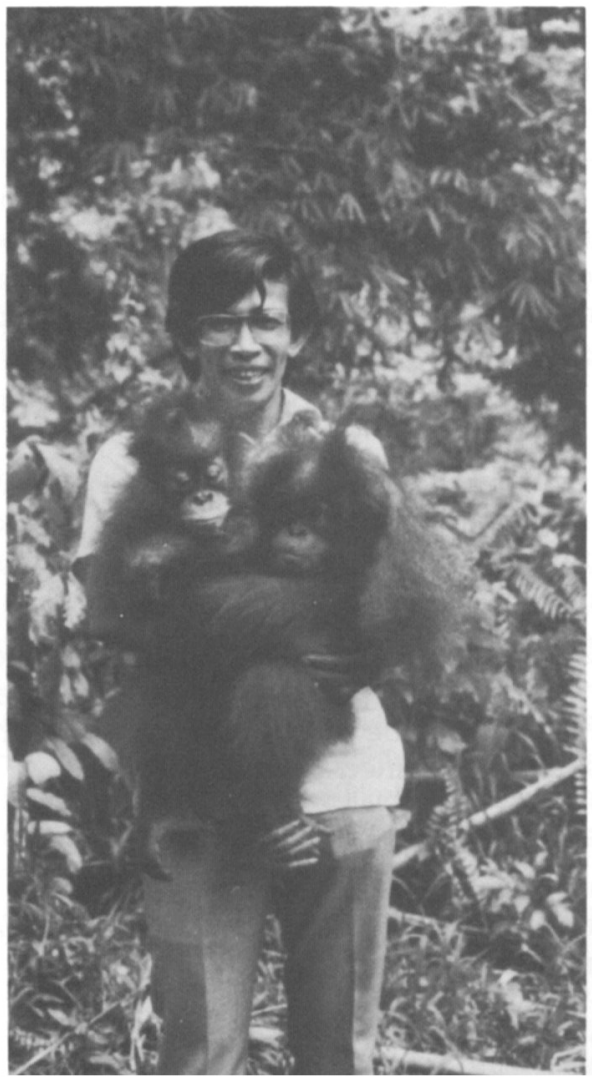

Rehabilitant orang-utans with a visitor at Bohorok, Sumatra, Indonesia. The rehabilitation centre concentrates on public education as well as returning apes to the wild, most of which are confiscated pets (M. Kavanagh). 
other reason, and moving the population to another area may seem to be a way of saving it. This may seem to be all the more important if it is the only population of a species or sub-species.

2.1.2 A captive population may be 'surplus', thus affording an apparent opportunity to restock the wild.

2.1.3 The existence of an apparently suitable habitat area may seem to offer a 'vacant lot' that could be used to accommodate an additional (or even the only) wild population of a particular species or sub-species.

2.1.4 At times, a small and isolated population may be thought to be seriously threatened by inbreeding, which the introduction of new conspecifics could relieve.

\subsection{Education reasons}

The establishment of an unrestrained animal population in a particular area may create an opportunity to bring people and the free-ranging animals into contact, and this may be exploited for educational purposes.

\subsection{Commercial reasons}

2.3.1 These may be the same as for education, except that the opportunity may be exploited for tourism. Education and commerce might thus be combined.

2.3.2 A supply of animals might be required for sale for research purposes, and a population might therefore be established with the aim of providing a sustained-yield harvest.

\subsection{Scientific reasons}

Behavioural research may sometimes be advanced by the artificial introduction of strange animals to one another.

\subsection{Compassionate reasons}

Animals may be translocated in the belief that the individuals concerned would be better off in the sense of being happier or suffering less than would otherwise be the case.

\subsection{Other reasons}

Both religious tradition and national pride have 136 motivated people to attempt the translocation of animal populations.

\section{Problems}

\subsection{The use of resources}

Any translocation operation requires the use of resources and, in most cases, large amounts of money and professional expertise will be used. It is therefore the planner's responsibility to demonstrate that the exercise in question is the best use of these resources in the pursuit of the overall goal of the operation (e.g. species conservation, public education, etc.).

\subsection{Local permission}

The permission of the local authorities will be necessary before any animal can be released, and in order to allow for any follow-up operations. This must be secured before any irrevocable commitment is made concerning the capture or dispensation of animals. In particular, alternative management options for the animals concerned should not be abandoned before permission has been granted (and all other obstacles have been overcome).

\subsection{Capture}

In the case of the translocation of a free-ranging population, the capture operation may be: stressful; fatal to some individuals; and/or require new techniques. A feasibility study by experienced professionals and with veterinary supervision will therefore be necessary. Preliminary planning may be helped by a review of relevant literature (e.g. Brett et al., 1982; Brockelman and Kobayashi, 1971; Harthoom, 1976; Seago, 1974).

\subsection{Transport}

Transport can be extremely stressful, and all arrangements should be made and carried out under experienced veterinary supervision. For species such as macaques, baboons and vervets that are commonly traded, guidelines exist which will help to minimise physical and psychological stress (e.g. Graham-Jones, 1974; Nolan, 1975). For rarer species there may be no body of experience upon which to draw, and the transporter will have to create transport Oryx Vol 17 No 3 
conditions that provide the optimum in: food; water; fresh air; temperature; protection against escape; visual inspection by the accompanying veterinarian; protection against injury of one animal by another; protection against cruelty by humans; companionship of other animals (for some species); and maternal care for infants. It should be recognised that pregnant females and very young infants are most likely to suffer during transportation but that all animals will benefit in proportion to the speed of the operation. Careful planning will minimise delays.

\subsection{Release}

The immediate release of new arrivals at their destination may result in panicked, disorientated and possibly unhealthy animals fleeing into an unfamiliar environment. This would: place additional stress upon the animals; rule out veterinary care for those that need it; increase the chance of accidental injury; increase the likelihood that individuals will become permanently lost from their groups and infants lost from their mothers; increase the risk of predation; and increase the chance that animals will flee into inappropriate areas, such as farmland if a release-point is at the (accessible) edge of a suitable habitat.

The release of translocated animals requires facilities where they may be settled down and maintained in a healthy state while they become familiar with their new surroundings. This procedure also allows for a quarantine period at the end of which the holding cages should be opened quietly, preferably when the animals are least active, so as to encourage them to emerge and explore in a gradual fashion. It will be more difficult to keep the animals calm if persons with whom they are not familiar are present when they are released. The temptation to turn the release into a media or political event should therefore be resisted.

\subsection{Rehabilitation}

In the special case of animals, particularly young animals, that have been in captivity, they may not be able to fend for themselves in the wild without a lengthy learning period. This will require the services of an extraordinarily dedicated person (or persons) for a period that may be as long as several years.

Can translocation help wild primates?

\subsection{Health}

3.7.1 Inter-population variation: animals from one natural population may differ from conspecifics of another, even nearby, population in disease characteristics. Thus the transfer of even one individual between two populations poses the risks of either introducing into the host population a disease against which its members have no resistance, or introducing the translocated animal to diseases against which it has no resistance.

3.7.2 Human contact: Old World primates especially are particularly susceptible to human diseases, so that the release of an animal that has had human contact into a population that has remained remote from people poses the risk of introducing human diseases to the latter. This is true even if the only contact that the introduced animals has had with humans was during the actual translocation process.

3.7.3 Stress: this may lower disease resistance, so producing unhealthy animals in an otherwise healthy population. Sub-clinical diseases may therefore become a definite hazard to the population purely as a result of stress.

\subsection{Environmental carrying capacity}

3.8.1 Conspecifics absent: if the species to be translocated is absent from the receiving area, it may be that the environment cannot support a population of the animal concerned, even if there was a population there in the past. It is therefore necessary to know why the species is absent before making the introduction and to decide whether or not it would be likely to find adequate resources for long-term survival if introduced or re-introduced. Re-introductions might be thwarted, for example, by a change in plant species-composition having eliminated essential food sources, or by the widespread effects of a change in water-table level. Plans for both introductions and re-introductions must take into account estimated worst years and seasons, not averages.

3.8.2 Conspecifics present: if the species to be translocated is already present in the receiving area, it must either be at the carrying capacity of the environment or held below that level by a 
high rate of mortality. Hunting and trapping are the most likely causes of the latter. In either case, it is difficult to see how the introduction of more animals could raise the population level in the long-term without there being a change in the environmental circumstances (such as the suppression of hunting), which would achieve the same result without the translocation. The only exception to this would be in the case of the resident population being so small as to be nonviable, yet the circumstances can be changed to enhance the viability of the combined residenttranslocated population.

\subsection{Hunting}

Before animals are translocated into an area, an investigation into local hunting practices is required. This is to eliminate the possibility that the translocation will do no more than provide short-term benefits to the local hunters, resulting in rapid extinction of the introduced population. If it is found that the local inhabitants do hunt, it may be possible to persuade them to leave the translocated animals alone.

\subsection{Hybridisation}

Where translocated animals are likely to come into contact with resident conspecifics, the introduction of individuals of a race or subspecies other than that of the residents will result in the artificial formation of a hybrid race. Most biologists would regard this as undesirable in view of the lengthy process by which sub-species are formed, and the possibility that differences between sub-species are functional, however subtle those differences may be.

\subsection{Social disruption of residents}

Primates form complex social communities and, although no such case has yet been documented, in some species the introduction of strangers might disrupt the resident community to the point where it would decrease rather than increase its growth. This possibility must at least be considered.

\subsection{Ecological disruption}

If conspecifics are absent from the receiving area, the introduction of the new species runs the 138 very real risk of creating a major ecological disturbance. Whilst primates are not likely to build up their numbers to pest proportions in a new natural habitat, the newly-arrived species might well out-compete and eliminate a competitor, and this might have ramifications throughout the community. Equally, there are many primates that could eliminate other species by predation, for instance by preying on eggs and nestlings. Furthermore, large herbivorous species such as the gorilla may have an especially significant impact upon vegetation, and hence upon the community at large.

\subsection{Long-term protection}

The translocated population will require longterm protection which goes beyond the granting of local permission; it must be guaranteed in such a way that it cannot be revoked with a change of mind or personnel at the office of the local wildlife authority.

\subsection{Finance}

Translocation is expensive: the recent transfer of some 200 Barbary macaques from France to Morocco, cost about $£ 100$ per monkey (Merz, unpublished). It is essential that the budget includes a substantial contingency fund. Even with the best possible planning, unexpected expenses are bound to arise.

\section{Conclusions}

There are so many problems inherent in translocation that it should not normally be attempted, and never without meticulous planning and adequate funding. An exhaustive feasibility study should precede the operation.

\subsection{Suitable cases}

The special cases in which we consider translocation to be a viable and constructive option are:

(a) The re-introduction of a rare species or subspecies to its former habitat if the animal in question was recently completely eliminated by hunting or some other human factor and if it can be guaranteed long-term protection. The basis for such a move must be to avoid the species 'having all its eggs in one basket', particularly if it is extinct Oryx Vol 17 No 3 
in the wild or known only in threatened locations. This is the only case in which animals might be translocated into a natural community that is itself independently of conservation interest.

(b) The introduction or re-introduction of animals, for conservation, educational, commercial, scientific, compassionate or other reasons, to sites where they will not come into contact with conspecifics and where the recipient ecological community either is not of particular conservation interest because it is common or degraded, or might not be conserved without the justification of protecting the translocated animals. Thus, whatever happens, the translocation exercise will not bring about the destruction of a viable natural community.

\subsection{Independent appraisal}

Each translocation operation should be thoroughly and independently refereed in the light of all the priorities and problems.

\subsection{Translocation and conservation strategy}

In view of the limited number of special cases in which translocation could contribute significantly to the survival of a species, and the many limitations of the technique in other respects, we believe that governments should be discouraged from using support for translocation projects as a 'soft option' to demonstrate their commitment to conservation. The real business of conservation is to do with the protection of habitats and the species within them. The role of translocation lies in the context of a fully developed national park system in each country, and is of little importance outside it.

\section{Acknowledgments}

We wish to thank the following people for their valuable comments and discussions: members of the Conservation Working Party of the Primate Society of Great Britain; participants in the International Conference for the Conservation of the Barbary Macaque, Gibraltar 1982; DrW. Angst; Dr D.J. Chivers; Mr J.E. Fa; Mr R.J. Hammond; Dr A.H. Harcourt and MrA. Mence.

\section{References}

Anon. 1968. Problems in species' introductions. IUCN Bull. 2(7), 70-71.

Anon. 1979. Reintroduction hazards. Oryx, 15, 80.

Brett, F.L., Turner, T.R., Jolly, C.J. and Cauble, R.G. 1982 Trapping baboons and vervet monkeys from wild, freeranging populations. J. Wildl. Mgmt, 46,(1), 164-174.

Brockelman, W.Y. and Kobayashi, N.K. 1971. Live capture of free-ranging primates with a blowgun. J. Wildl. Mgmt, 35(4), 852-855.

Campbell, S. 1980. Is reintroduction a realistic goal? In: Conservation Biology: an evolutionary-ecological perspective, pp. 263-269 (Eds M.E. Soulé and B.A. Wilcox). Sinaur Sunderland, Massachusetts.

Grahame, I. 1980. Reintroduction of captive-bred Cheer pheasants. Int. Zoo Yb. 20,36-40.

Graham-Jones, O. 1974. Some aspects of air transport of animals. Int. Zoo Yb. 14, 34-37.

Harthoorn, A.M. 1976. The Chemical Capture of Animals. Baillière Tindall, London.

Merz, E. (unpublished) Short report on the reintroduction of four groups of Macaca sylvanus from two French enclosures to the Middle Atlas of Morocco in 1980.

Nolan, M.A. 1975. Importing primates for research. In: Primate Utilization and Conservation, pp. 15-19 (Eds G. Bermant and D.G. Lindburg). Wiley (Interscience), New York.

Seago, J. 1974. Responsiblities of an animal catcher. Int. Zoo Yb. 14, 39-40.

J. O. Caldecott, Sub-Department of Veterinary Anatomy, University of Cambridge, Tennis Court Road, Cambridge, UK.

M. Kavanagh, IUCN Conservation Monitoring Centre, 219c Huntingdon Road, Cambridge, UK. 